\title{
An unusual facial ulcer
}

\author{
Amanda Abramson, MD, and Alan Menter, MD
}

A

69-year-old Caucasian man presented with a lesion on his forehead which "grew overnight" (Figure 1). It was located at the edge of a scar of a previously removed squamous cell carcinoma. The patient has had numerous squamous cell carcinomas and mild hyperlipidemia. What is your diagnosis?

Over the ensuing week, the patient developed two fresh ulcers on his forehead "overnight" (Figure 2). There was no erythema, flaking, pain, numbness, or tingling in the areas prior to the development of the ulcers. The two new lesions were tender. There was no known trauma. Now, what is your diagnosis?

\section{DIAGNOSIS: Herpes zoster.}

Herpes zoster is the reactivation of varicella zoster virus. It typically presents with a prodrome of pain, tingling, or tenderness, and then an eruption of grouped vesicles on an erythematous base occurs within a sensory dermatome. The most common complication is postherpetic neuralgia, which is a neuropathic pain syndrome that develops after the rash has resolved (1-3). It is typically described as burning, stabbing, or

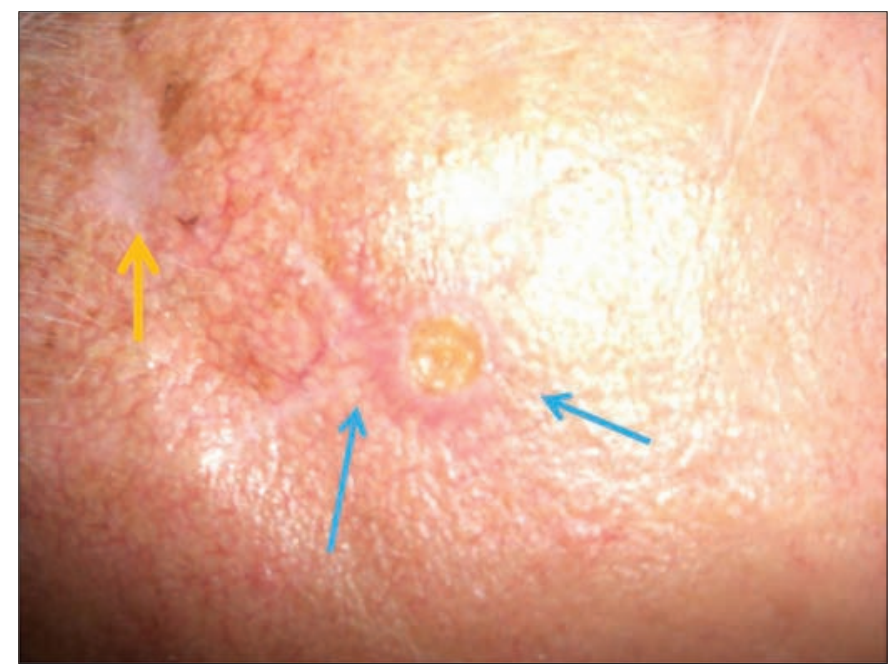

Figure 1. Right frontolateral forehead. A 5-mm well-circumscribed superficial ulcer with a rolled pink border and surrounding telangiectasias at the medial edge of a scar. Superolateral to the lesion, there is a second scar from a previously treated nonmelanoma skin cancer.

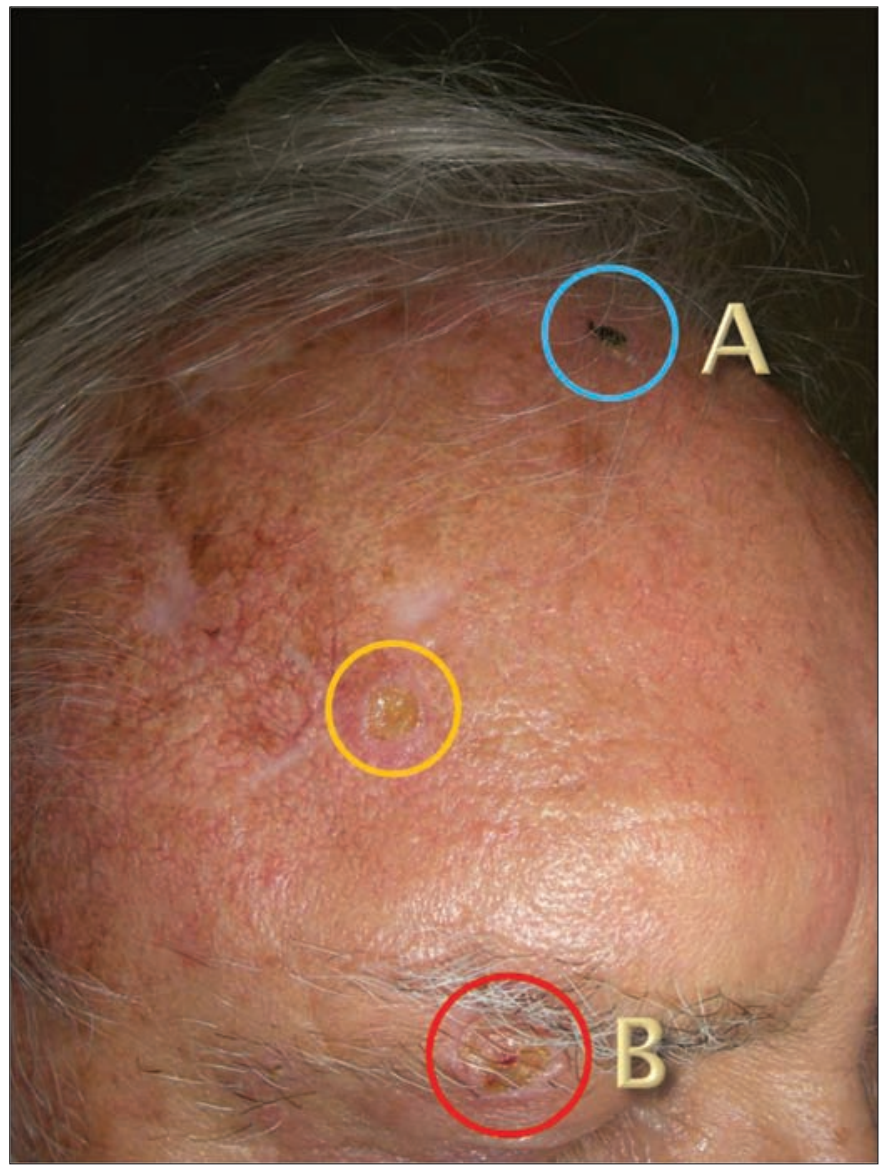

Figure 2. The blue circle (A) is a superficial ulcer with an overlying hemorrhagic crust along the scalp fringe to the right of the midline. The red circle $(B)$ is a superficial ulcer with a rolled border within the right eyebrow. The yellow circle indicates the primary lesion.

shocklike pain in the area where the rash was located $(1,4)$. Postherpetic neuralgia occurs in $14 \%$ to $20 \%$ of patients with herpes zoster (2).

From the Division of Dermatology, Department of Internal Medicine, Baylor University Medical Center at Dallas.

Corresponding author: Amanda Abramson, MD, Division of Dermatology, Department of Internal Medicine, Baylor University Medical Center at Dallas, 3900 Junius Street, Suite 145, Dallas, Texas 75246 (e-mail: abramsonmd@ gmail.com). 
A vaccine for the prevention of herpes zoster and postherpetic neuralgia, Zostavax, was approved by the Food and Drug Administration in 2006 (5). It is a live virus vaccine composed of at least 19,400 plaque-forming units of the Oka/Merck strain of the varicella zoster virus. The potency of this vaccine is 14-fold higher than that used in children (2). Zostavax reduces the risk of developing herpes zoster by $51 \%$ and decreases the incidence of postherpetic neuralgia by $67 \%(3,4)$; further, it decreases the burden of illness of herpes zoster by $61 \%$ and decreases the duration of postherpetic neuralgia by $57 \%(3,4)$. Therefore, disease severity is decreased in patients who experience a herpes zoster outbreak despite vaccination $(3,4)$. Most recently, Zostavax was shown to decrease the risk of ophthalmic involvement, therefore decreasing the risk of serious vision-threatening sequelae (4). Zostavax is recommended by the Centers for Disease Control and Prevention for use in patients $>60$ years of age who are not immunocompromised $(3,4)$.

Our patient had received the Zostavax vaccine 2 weeks prior to his initial presentation. The patient is otherwise healthy and is not immunocompromised. To date, there are no reports of the Zostavax Oka/Merck varicella virus strain causing herpes zoster itself. However, there are reports of a herpes zoster-like reaction occurring at an average of 16 days after vaccination $(4,6)$. Wild-type varicella zoster virus DNA was isolated by polymerase chain reaction from the active lesions of the vaccinated patients with a herpes zoster-like reaction, confirming the reactions were likely not due to the vaccine (6). Thus, our patient experienced a herpes zoster-like reaction from the vaccine given 2 weeks prior to the onset of his facial lesions.

The case highlights the importance of conducting a thorough history, including a vaccination history. It exemplifies how a vaccine-dampened outbreak of herpes zoster can clinically mimic nonmelanoma skin cancer. Therefore, truncated herpes zoster should be included in the differential diagnosis for nonmelanoma skin cancer for recently vaccinated individuals; a biopsy of the lesion is necessary for confirmation of disease.

1. Oxman MN. Zoster vaccine: current status and future prospects. Clin Infect Dis 2010;51(2):197-213.

2. Sanford M, Keating GM. Zoster vaccine (Zostavax): a review of its use in preventing herpes zoster and postherpetic neuralgia in older adults. Drugs Aging 2010;27(2):159-176.

3. Harpaz R, Ortega-Sanchez IR, Seward JF; ACIP, CDC. Prevention of herpes zoster: recommendations of the Advisory Committee on Immunization Practices (ACIP). MMWR Recomm Rep 2008;57(RR-5):1-30.

4. Tseng HF, Smith N, Harpaz R, Bialek SR, Sy LS, Jacobsen SJ. Herpes zoster vaccine in older adults and the risk of subsequent herpes zoster disease. JAMA 2011;305(2):160-166.

5. Merck Sharp \& Dohme. Zostavax [package insert]. Whitehouse Station, NJ: Merck, 2006.

6. Simberkoff MS, Arbeit RD, Johnson GR, Oxman MN, Boardman KD, Williams HM, Levin MJ, Schmader KE, Gelb LD, Keay S, Neuzil K, Greenberg RN, Griffin MR, Davis LE, Morrison VA, Annunziato PW; Shingles Prevention Study Group. Safety of herpes zoster vaccine in the Shingles Prevention Study: a randomized trial. Ann Intern Med 2010;152(9):545-554. 